\title{
QUALITY IMPROVEMENT ATTEMPTS IN PRE-ANALYTICAL PHASE
}

\author{
Osman Sianipar \\ Department of Clinical Pathology and Laboratory Medicine, Faculty of Medicine, Public Health and Nursing Universitas Gadjah Mada, \\ Yogyakarta, Indonesia. E-mail: osmansianipar@ugm.ac.id.
}

\begin{abstract}
In clinical laboratory services, a pre-analytical phase has played an important role in term of quality and patient safety. The pre-analytical phase is a phase before analyzing a sample in a laboratory which includes patient preparation, sampling, labeling, sample transportation, sample storage, and sample preservation that might influence the laboratory results. This phase involves interaction between patient, doctor, laboratory personnels, and other staffs outside the laboratory. Many samples are collected, many laboratory tests are requested, many individuals are involved. Therefore, laboratory errors might occur during the pre-analytical phase. Laboratory errors can occur either in pre-analytical, analytical, or postanalytical phases; however, the most frequent errors occur in the pre-analytical phase. In this article, quality improvement attempts in the pre-analytical phase will be discussed to minimize pre-analytical error.
\end{abstract}

Key words: Quality improvement, laboratory service, pre-analytical phase, laboratory error

\section{INTRODUCTION}

The laboratory testing process is divided into the pre-analytical, analytical and post-analytical phases. The pre-analytical phase includes all activities and aspects of the clinical laboratory procedure that occurs before the analytical phase. The pre-analytical phase is the most susceptible step of the overall process of laboratory testing and is considered to be the greatest obstacles to the laboratory personnel. The quality of the pre-analytical phase influences the quality of the test results. However, pre-analytical activities, management of unsuitable specimens and reporting policies are not well standardized, and globally harmonized. ${ }^{1}$

The pre-analytical phase can be categorized into the"conventional" and pre-pre-analytical phase. The "conventional" pre-analytical phase is under the control of the laboratory involving a series of related processes starting with patient identification, the selection of ideal tubes, proper transportation and storage, and ended with the preparation of the samples. It also involves the processes required to make a proper sample for analysis such as centrifugation, aliquotting, diluting and sorting the specimens into batches for their introduction into an automated analyzer. Whereas pre-pre-analytical phase happens outside the laboratory and composes of the selection of appropriate tests based on the clinical question, ordering, collecting and handling, transportation and reception of samples before testing. ${ }^{2,3}$ Cycle of events in the pre-analytical stage includes test order, sampling, sample transportation, sample delivery, and sample processing. ${ }^{4}$

Standards associated with blood sampling, sample transportation and handling are available, but compliance to those guidelines is relatively low, specifically those outside the laboratory and when blood sampling is performed without the direct control of the laboratory personnel. Moreover, for some most critical procedures within the pre-analytical phase, recommended international guidelines, and related quality measures are not available yet. There is a huge variation in the global criteria for specimen rejection, the different strategies management of unacceptable samples, sample processing, and test results reporting. ${ }^{1}$

Laboratory errors can occur either in the pre-analytical phase, analytical phase, or post-analytical phase. Among these three phases, error in the pre-analytical phase is the most frequent. ${ }^{5,6}$ Pre-analytical errors cover up to $70 \%$ of all mistakes occurred in the clinical laboratory, most of which arise from problems in patient preparation, sample collection, transportation, and preparation for analysis and storage. ${ }^{5,7-9}$ The objective of this review is to discuss quality improvement attempts in the pre-analytical phase in order to improve the quality and patient safety in clinical laboratory 
services could be improved.

\section{DISCUSSION}

One of the activities in pre-pre-analytical phase is laboratory test ordering. The purpose of this activity is to confirm/determine treatment, to exclude/physician's uncertainty, and to reassure/patient's request. ${ }^{10}$ One of laboratory errors that can occur in this step is inappropriate prescription of serological tests for viral hepatitis $B$ and $C$ infection that accounts for $55.8 \%$ cases. It consists of $54.2 \%$ excessive, $0.5 \%$, insufficient, $0.7 \%$ invalid, and $0.4 \%$ imprecise prescriptions. ${ }^{11}$ It was also reported that for the in-patient population, there were $32 \%$.total errors of test order, particularly for unintelligible test orders. ${ }^{12}$

A laboratory error may occurr in the pre-analytical phase due to either inappropriate sample, inappropriate requisition form, inappropriate sample volume, or inappropriate tube. The inappropriate sample includes incorrect labeling, unlabeled, grossly haemolyzed sample, unsuitable sample for analysis, and old sample. inappropriate requisition form includes inaccurate/incomplete information, no test requested, and form a bloodstained. Sample leaked during transit, sample broken during transit, no specimen received, sample with insufficient quantity, and sample broken/spilled in the laboratory are categorized as sample with inappropriate volume. Lastly, sample collected in an appropriate blood tube is categorized as incorrect sample. Based on the type of sample, majority errors occurr in the blood sample. These errors result in rejection of the requisitions which are frequently dominated (92\%) by blood samples in clinical laboratory services. The second mostly found rejection is urine sample (5\%) followed by cerebrospinal fluid, feces and pleural fluid with the same number of $2 \%$. It was also reported in that study that total rejection rate during the period of $2015-2016$ was $2.1 \%$. The study also reported that the most commonly found error from 2012 until 2016 was unlabelled samples ranging between 21 - 44\% per year. Labeling error including incorrect labeled and unlabelled sample from 2015 to 2016 resulted in a rejection rate of $1.2 \%{ }^{13}$ Another study was performed at the clinical chemistry laboratory of tertiary care, 600 bed Govind Ballabh Pant Hospital, New Delhi, India reported total error of $1.52 \% .{ }^{14} \mathrm{~A}$ different study conducted at a 2185 bed hospital in Assam, India reported a higher error rate of $5.2 \%{ }^{15}$
Laboratory errors may result in a mild, moderate, severe, or highly severe impact on patient care. ${ }^{5}$ Therefore, attempts should be done to minimize an error rate and its impact on the patient's care. It can be achieved by the implementation of a quality improvement program. The program should be able to identify the source of error, the procedure to prevent an error, develop quality indicators, and it needs to be regularly evaluated based on quality indicator-related achievement. The quality indicators in pre-analytical phase are composed of 8 domains, namely appropriateness of test request, patient identification, purpose of request, order entry, sample identification, sample collection, sample transportation, sample acceptance/ rejection. ${ }^{9}$

In order to improve efficiency and patient safety, The American Society of Clinical Pathologist recommend the selection of appropriate laboratory test for right patient at right time such as: population-based screening is not allowed for 25-OH-Vitamin D deficiency; low-risk Human Papillomavirus (HPV) testing is not allowed; routine preoperative testing for low-risk surgeries without a clinical indication must be avoided; methylated septin (SEPT9) is only ordered on patients for whom conventional diagnostics are not possible; and the use of bleeding time tests are not allowed to guide patient care. These recommendations are given because these tests are frequently ordered previously in order to use the laboratory resources more efficiently. ${ }^{16}$ Another thing that should be considered is that the test order along with the patient identification must bewritten in either paper or electronic test order completely. Furthermore, patient preparation is required in this step. Patient should be informed about the test requirement before laboratory test is performed. For example, overnight fasting is required for analytes test like glucose and cholesterol and sample collection at two different times in a day is required for diurnal variation of analytes such as cortisol and adrenocorticotropin. ${ }^{3}$

Patient should also be accurately identified during the sample collection. It is important to identify a patient properly to ensure that the sample is drawn from the right patient. Laboratory personnel or nurse who takes the sample should verify patient identification by observing an identification band especially for in-patient before the sample is taken. Drawing sample from the wrong patient or incorrect labeling of the sample will give rise to incorrect results and subsequently may affect the decision of 
patient care. It may also cause repeated sampling and testing and results in unnecessary addition to the cost for the care. ${ }^{3}$ The use of bar-coded wristband, patient identification by at least two personnels when taking samples, use of biometric information such as fingerprint or iris scanning, checking requisition and the results, labelling sample container immediately after collection are possible attempts to prevent patient/sample misidentification. ${ }^{17}$

In the step of sample collection, errors might occurr. It was reported that error rate due to "hemolysis sample", "urine sample not submitted" and "clotted sample"were $8.76 \%, 1.66 \%$, and $1.41 \%$, respectively. ${ }^{18}$ Samples which are sent in a clinical laboratory might be rejected. It was reported that the most common causes for rejection were unlabelled samples $(37 \%)$, wrongly labeled specimens $(23 \%)$, use of incorrect sample collection tube (14\%) and incomplete or inaccurately completed test order forms $(14 \%) \cdot{ }^{13}$ Another study reported that rejection rates of hemolysis, clotted specimen and insufficient volume of sample error were $8 \%, 24 \%$, and $34 \%$, respectively. ${ }^{12}$ Filling blood collection tube up to its required volume is an attempt to prevent insufficient sample volume. Clotted plasma sample could be prevented by following manufacture's guideline for tube mixing. ${ }^{17}$

Hemolysis may occur due to either alcohol contamination or an excessive probing and/or "fishing" to find a vein when drawing the blood sample. It can give rise to the false high result of analytes such as potassium, lactate dehydrogenase (LD), iron and magnesium. ${ }^{3}$ False laboratory test results also occur due to time and site to apply a tourniquet. The tourniquet should be applied 3"- 4" above the venipuncture site and should be applied on the arm for no more than one minute. Prolonged application of tourniquet may result in false high test result especially for critical analytes such as calcium. Sufficient volume of the sample should be taken. ${ }^{3}$ Avoiding vigorous mixing/agitation of blood sample, avoiding the use of tourniquet for more than one minute, use of closed system of blood sampling, use of transfer device to transfer blood from the syringe, use of Luer lock access device and discarding tube when drawing from the line are attempts to prevent hemolysis. ${ }^{17}$

After collection of the samples, at least other three preanalytical phases such as transport, centrifugation, and storage that must be carefully taken care to prevent deterioration namely. Ideally, the collected sample should be transported immediately in non-refrigerated and ambient temperature $\left(15-22^{\circ} \mathrm{C}\right)$ to a clinical laboratory for further process. Pneumatic tube, robot, or courier can be used for sample transport. Transport by courier, especially for a long time or under extreme temperature conditions, may affect unstable clotting factors such as factor $V$ and VIII. For in-patients, transport of sample using a pneumatic tube system may result in hemolysis. ${ }^{19}$ The sample has to be transported using an appropriate transport container and regular monitoring of the container condition such as temperature and humidity. Several other attempts related to sample transportation are as follows: direct sample collection in a laboratory; confirmation of sample identification before transport. monitoring safety conditions and stability in proper condition; a whole blood sample in the tube remain capped for transport immediately after collecting; samples transfer in vertical position; use of Pneumatic Tube Systems (PTS) for the transport of samples for platelet function analysis purposes should be avoided; and proper control of temperature at which samples are kept for analysis. ${ }^{20}$ In this step, error that might occurr is prolonged transportation. It was reported that error due to inconvenient transport conditions accounted for $0.005 \%{ }^{12}$

The sample of cerebrospinal fluid should be sent to the laboratory immediately. Delayed transportation of this sample can cause the death of organisms and also cause the disintegration of leukocytes. If delayed transportation can be anticipated, the sample should be kept at room temperature, since refrigeration tends to kill $\mathrm{H}$. influenza. ${ }^{3}$

After all the processes of sample transportation are accomplished, the sample should be processed properly before analysis. The processes include centrifugation and separation or aliquot preparation, preservation or storage, freezing and thawing, pipetting and sorting, or incubation sample in enrichment media. Centrifugations and separations are mostly required for analysis of chemistry, serology, and hemostasis. Blood samples from patients who are under anticoagulant treatment may take longer time to clot. Blood sample in the tubes should be let to clot at room temperature and kept in the rack vertically with the tubes closed. Centrifugation too early may cause a gelatinous and/or fibrinous serum sample that will require re-centrifugation. Plasma specimens are obtained from the blood by mixing and immediate centrifugation to reduce the turn-around-time for test results. Serum and plasma tubes without gel can 
be centrifuged at 1,000 relative centrifugal force (RCF) for ten minutes. After separation, the serum is stable at room temperature for eight hours, and up to 48 hours if kept at $2-4^{\circ} \mathrm{C}$. The serum should be frozen at $-20^{\circ} \mathrm{C}$ in an aliquot tube after 48 hours. Photo-sensitive analytes such as bilirubin, need to be protected from light and the stability can be maintained by wrapping the tube in aluminum foil. ${ }^{3}$

Preservation of urine sample is required if it can not be immediately processed immediately after it arrives at the laboratory. When a preservative is needed, it should be added to the sample container before the urine collection starts. Urine sample buffered with boric acid may reduce the harmful effects to the organism. ${ }^{3}$

These following attempts should be made during centrifugation for hemostasis analysis: use of temperature-controlled centrifuge for processing routine coagulation assays; validatione of the centrifuge every 6 months or after modifications before use in order to assure that Platelet-Poor Plasma (PPP) is achieved; monitoring the absence of vibration (during acceleration/deceleration processes) due to lack of centrifuge maintenance; centrifugation of the primary tube for coagulation testing at $1500 \mathrm{~g}$ for 15 minutes. In case of emergency, for Prothrombin Time (PT), Activated Partial Thromboplastin Time (APTT) and fibrinogen, fresh plasma with higher centrifugation force (greater than $1500 \mathrm{~g}$ ) and shorter time (less than 10 min) can be used; the preparation of PPP requires a double centrifugation to obtain a residual platelet count lower than $10 \times 109 / \mathrm{L}$; following initial centrifugation, the plasma must be transferred carefully to a nonactivating plastic centrifuge tube using an automatic pipette, and recentrifuged for about $15 \mathrm{~min}$. The preparation of Platelet-Rich Plasma (PRP) for platelet function analysis requires a centrifugation at $200-250 \mathrm{~g}$ for 10 minutes without application of a rotor brake. ${ }^{20}$

Some attempts regarding the sample storage for hemostatis assay are as follows: samples must be stored at room temperature $\left(15-25^{\circ} \mathrm{C}\right)$ until analysis, whole blood assays must be performed less than 4 hours after blood sampling and centrifugation less than 1 hour, extreme temperature (e.g. refrigerated or high) should be avoided, PPP must be stored at room temperature $\left(15-25^{\circ} \mathrm{C}\right)$ or at $-80^{\circ} \mathrm{C}$ until analysis, if the whole blood sample is centrifuged within 1 hour of collection, the plasma can be left on top of the cells at room temperature up to 4 hours prior to testing; Time from sampling to analysis depends on analyte such as samples for PT or
International Normalized Ratio (INR) have longer stability (24h) at room temperature, samples for APTT should be performed using fresh plasma less than 4 hours (less than 1 hour in patients treated with unfractionated heparin). For platelet function assays, samples should be incubated at room temperature for at least $15 \mathrm{~min}$ before analysis. Testing should be completed less than 3-4 hours of collection. F actors $V$ and VIII analyses should be performed less than 3 hours. Fibrinogen, protein $C$, and antithrombin activity appear to remain relatively constant when stored at room temperature for up to 7 days. Protein $\mathrm{S}$ activity is unstable, with a statistically significant loss of activity at 8h. Von Willebrand factor (VWF) appears to be stable at room temperature for 48 hours. $^{20}$

Attempts regarding freezing and thawing for hemostasis assay are as follows: Frozen samples are now allowed to be used for PT, APTT and factor VIII tests; centrifugation and plasma aliquot freezing are required for samples that are not tested within 4 hours; use of rapid freezing technique (liquid nitrogen); samples are preferably be stored at $-70^{\circ} \mathrm{C}$ (or below) rather than $-20^{\circ} \mathrm{C}$. Plasma samples were frozen at $-20^{\circ} \mathrm{C}$ remain stable for 2 weeks, while plasma frozen at $-80^{\circ} \mathrm{C}$ remains stable for $6-18$ months depending on the parameter. re-freezing samples are not allowed (preparation a sufficient number of aliquots is required). Rapid samples thawing at $37^{\circ} \mathrm{C}$ at least $5 \mathrm{~min}$ in a water bath at $37^{\circ} \mathrm{C}$ not at room temperature, on a bench or in a microwave oven followed by immediate testing is to prevent denaturing fibrinogen. After thawing, the sample must be gently mixed to resuspend any cryoprecipitate. Use of vortex and shaking are not allowed. ${ }^{20}$

A particular training activity to the hospital nursing staff and nursing students who were responsible for blood sampling was able to reduce proportion of non-conforming samples. There was reduction of non-conforming sample from $0.29 \%$ in 2007 to $0.07 \%$ in 2015 , resulting in $75.86 \%$ improvement. It was also reported that an error in sample identification reduced by $0.056 \%$ and led to 96.55\% improvement. A positive impact of this intervention was a $127.84 \%$ increase in the total number of specimens received. ${ }^{21}$

\section{CONCLUSION}

The pre-analytical phase is the phase before the clinical sample processing for further analysis. It can be extra laboratory which is also known as 
pre-analytical phase and intra laboratory. It includes test order, sample collection, sample transport, sample delivery, and sample processing. It is known that most laboratory errors occurr during this phase. These errors might have an impact (which are categorized to be mild, moderate, severe, and very severe) on patient care. Therefore, quality improvement attempts have to be taken in order to improve the quality of service and patient safety. Quality indicator-related achievement should be done evaluated regularly by measuring quality indicators and conducting corrective actions on the basis of route cause.

\section{REFERENCES}

1 Simundic A, Lippi G. Preanalytical phase - a continuous challenge for laboratory professionals. Biochem Med, 2012; 22(2): 145-149.

2 Rin G Da. Pre-analytical workstations: A tool for reducing laboratory errors. Clin Chim Acta, 2009; 404(1): 68-74.

3 Neogi SS, Mehndiratta M, Gupta S, Puri D. Pre-analytical phase in clinical chemistry laboratory. J Clin Sci Res, 2016; 5(3): 171-8.

4 Chhillar N, Khurana S. Effect of Pre-analytical errors on quality of laboratory medicine at a Neuropsychiatry Institute in North India. Ind J Clin Biochem, 2011; 26(1): 46-49.

5 Bonini P, Plebani M, Ceriotti F, Rubboli F. Errors in laboratory medicine. Clin Chem, 2002; 48(5): 691-698.

6 Carraro P, Plebani M. Errors in a stat laboratory: Types and frequencies 10 years later. Clin Chem, 2007; 53(7): 1338-1342.

7 Lippi G, Chance JJ, Dazzi P, Fontana R, Grankvist K, Huisman $W$, et al. Preanalytical quality improvement: From dream to reality. Clin Chem Lab Med, 2011; 49(7): 1113-1126.

8 Cornes MP, Atherton J, Pourmahram G, Borthwick H, Kyle B, West J. Monitoring and reporting of preanalytical errors in laboratory medicine: The UK situation. Ann Clin Biochem, 2016; 53(2): 279-284.

9 Plebani M. Quality Indicators to detect pre-analytical errors in laboratory testing. Clin Biochem Rev, 2012;
33(3): 85-88.

10 Houben PHH, Winkens RA, Weijden van der, Vossen RCRM, Naus AJM, Grol RPTM. Reasons for ordering laboratory tests and relationship with frequency of abnormal results. Scand J Prim Heal Care, 2010; 28(1): 18-23.

11 Galula G, Buffet C, Robba L, Poissonet M. Assessment of prescription practices for serological tests for viral hepatitis B and C in the Greater Parisian area in 2002. Gastroenterol Clin Biol, 2006; 30(4): 517-524.

12 Atay A, Demir L, Cuhadar S, Saglam G, Unal H, Aksun S, et al. Clinical biochemistry laboratory rejection rates due to various types of preanalytical errors. Biochem Med 2014; 24(3): 376-382.

13 Tapper MA, Pethick JC, Dilworth LL, Mcgrowder DA. Pre-analytical Errors at the Chemical Pathology Laboratory of a teaching hospital. J Clin Diagn Res, 2017; 11(8): 16-18.

14 Chawla R, Goswami B, Tayal D, Mallika V. Identification of the types of preanalytical errors in the clinical chemistry laboratory: 1-year study at G.B. Pant Hospital. Lab Medicine, 2010; 41(2): 89-92.

15 Begum F. A study of pre-analytical errors in a hospital-based clinical biochemistry laboratory. IntJ Bioassays, 2014; 3(9): 3270-3275.

16 Hilborne L. Choosing wisely: Selecting the right test for the right patient at the right time. MLO Med Lab Obs, 2014; 46(5): 40.

17 Kaushik N, Green S. Pre-analytical errors: Their impact and how to minimize them. MLO Med Lab Obs 2014; 46(5):22, 24, 26.

18 Rivas-Ruiz F, Molina-Mendoza P. Pre-analytical errors management in the clinical laboratory: A five-year study. Biochem Med, 2014; 24(2): 248-57.

19 Sodi R, Darn SM, Stott A. Pneumatic tube system-induced hemolysis: Assessing sample type susceptibility to hemolysis. Ann Clin Biochem, 2004; 41(3): 237-240.

20 Magnette A, Chatelain M, Chatelain B, Cate H Ten, Mullier F. Pre-analytical issues in the hemostasis laboratory: Guidance for the clinical laboratories. Thromb J, 2016; 14: 1-14.

21 Al-Ghaithi H, Pathare A, Al-Mamari S, Villacruris R, Fawaz N, Alkindi S. Impact of educational activities in reducing pre-analytical laboratory errors. Sultan Qaboos Univ Med J, 2017; 17(3): 309-313. 